but when they present with significant complication, surgery is warranted. Cholecystocolonic fistulas are uncommon and when present is usually associated with significant biliary disease. This paper aims to present an evenly atypical manifestation of this variant and much more that it presents in a very rare manner in the form of lower GI Bleeding.

Methods We present a case of a 61-year-old male with Acute Myelogenous Leukaemia with no established biliary disease. $\mathrm{He}$ was post-appendectomy with loop ileostomy. He complained of recurrent abdominal pain with hematochezia.

Results Plain film of the abdomen revealed gas distended bowel loops in the disorganised pattern. Initial colonoscopy prior to the contemplated takedown of ileostomy was done revealing an ascending colon stricture and colitis. Anaemia and infection were addressed. The abdominal pain persisted, and he had massive hematochezia, 2nd look colonoscopy revealed an ulcerated area oozing with blood and bile flowing into the ascending colon hence a fistula was suspected. The patient did not respond to conservative management of the bleeding hence he underwent Right hemicolectomy and cholecystectomy with adhesiolysis and takedown of ileostomy which confirmed the diagnosis of cholecystocolonic fistula and was further supported by the histopathologic findings.

The patient improved post-operatively and was discharged subsequently.

Conclusions Be prudent in handling atypical scenarios in a morbid and complicated patient. A stepwise approach and a high index of suspicion especially in unusual situations can often lead us to the correct diagnosis.

\section{IDDF2018-ABS-0255 IMPACT OF COMMENSAL HUMAN GUT MICROBIOTA IN THE EMERGENCE OF EXTENSIVE DRUG-RESISTANT PATHOGENS}

${ }^{1}$ Sayantan Banerjee*, ${ }^{2}$ Satyabrata Bag, ${ }^{2}$ Jyoti Verma, ${ }^{2} \mathrm{D}$ Anbumani, ${ }^{2}$ Bhabatosh Das. ${ }^{1} \mathrm{All}$ India Institute of Medical Sciences, New Delhi, India; ${ }^{2}$ Centre for Human Microbial Ecology, Translational Health Science and Technology Institute, NCR Biotech Science Cluster, Faridabad, India

\subsection{6/gutjnl-2018-IDDFabstracts.43}

Background Antimicrobial resistance (AMR) in human health associated bacterial pathogens is a serious concern across the globe. Human gastrointestinal tract (GIT) is populated with trillions of microbial species that contribute to nutrient assimilation, host and microbial cell signalling, pathogenesis and antimicrobial resistance (AMR). Indiscriminate usages of antimicrobial in healthcare, livestock, and agriculture provide an evolutionary advantage to the resistant variants. The ascendency of resistant variants threatens the efficacy of most, if not all, of the antimicrobial drugs commonly used to prevent or cure microbial infections. The aim of this current study was to understand the impact of commensal gut microbiota in the emergence of extensively drug-resistant (XDR) enteric pathogens.

Methods Commensal anaerobic gut microbiota was isolated from the healthy Indians without a history of hospitalisation and antibiotic intake in last one year. AMR phenotypes were characterised in an anaerobic workstation using commercially avail Epsilometer test (E-test) strip. Genome of all the isolates was sequenced in GS-FLX + pyrosequencer. Genome assembly was done in GS de novo assembler. Genome annotation and analysis of resistance genes were done using Rapid Annotations using Subsystems Technology (RAST) platform.

\section{Results}

i. AMR traits in enteric bacteria, both in commensals and pathogens, are physically linked with mobile genetic elements (MGEs) and could rapidly disseminate to the bacterial species through horizontal gene transfer (HGT).

ii. Among commensal gut microbiota, the highest number of resistance phenotypes $(n=12)$ and AMR encoding functions were detected in Faecalibacterium prausnitzii.

iii. Among pathogens, Vibrio cholerae and Klebsiella pneumoniae showed maximum resistance phenotypes $(n=22)$.

iv. Genes encoding antibiotic resistance in commensal and pathogenic bacteria are physically linked with MGEs and could disseminate vertically to the progeny and laterally to the distantly related microbial species.

Conclusions XDR pathogenic and commensal enteric bacteria are isolated from healthy Indian subjects without a significant history of hospitalisation or antibiotic consumption. Consequently, the transmissible AMR genes present in the genome of gut commensals could be a potential source of resistance functions for the enteric pathogens.

\section{IDDF2018-ABS-0264 PREVALENCE OF ANXIETY, DEPRESSION AND QUALITY OF LIFE IN AMONG SCHOOL GOING ADOLESCENT WITH IRRITABLE BOWEL SYNDROME}

Nitin Kumar*. Department of Basic and Applied Biology, Vivekananda Global University, Jaipur, Rajasthan, India

\subsection{6/gutjnl-2018-IDDFabstracts.44}

Background Irritable bowel syndrome (IBS) is the most prevalent functional gastrointestinal disorders (FGIDs) that affects different aspects of life and patients experienced depression and anxiety more than others. There have been few Asian studies regarding anxiety and depression associated IBS. The aim of this study was to evaluate the frequency, magnitude and importance of anxiety and depression in among school going adolescent with IBS in Jaipur city, India.

Methods A Cross-sectional survey of students of four classes from 9th to 12th studying in government schools in Jaipur city, India. Ten government schools in Jaipur were randomly selected through lottery method. In each school, for each of the four classes, a section was randomly selected again by the lottery method. Forty students were selected from each school reaching a sample size of 470 . This clinical trial study was done in IBS patients (with mild-to-moderate symptoms) divided into two case and control groups. All participants were asked to complete self-administered questionnaires: one addressing symptom severity and the hospital anxiety and depression scale (HADS). The patients were also asked to complete the IBS-specific quality of life (IBS-QOL) questionnaire.

Results Anxiety and depression were observed in $32.1 \%$ and $34.5 \%$ of IBS patients, respectively, and in $26.6 \%$ and $17.2 \%$ of healthy subjects, respectively $(\mathrm{p}<0.05$ for both) in school adolescent. Both anxiety and depression were associated with self-reported symptom severity $(\mathrm{p}<0.05$ and $\mathrm{p}<0.05$, respectively). As determined by multivariate analysis, symptom severity was the most important factor in the prediction of anxiety and depression. Self-reported symptom severity and 
depression were clearly and independently associated with the overall IBS-QOL score.

Conclusions Anxiety and depression were frequently observed in school going adolescent in Jaipur city IBS patients and were related to the severity of their symptoms and the impairment of the patient's QOL. Our data suggest that assessing anxiety and depression is important when evaluating IBS patients. There is a need for early and effective identification of anxiety, depression that can prevent many psychiatric disorders at their nascent stage with irritable bowel syndrome.

\section{Basic Hepatology}

\section{IDDF2018-ABS-0039 0-GLCNACYLATION ON RAB3A ATTENUATES ITS EFFECTS ON MITOCHONDRIAL OXIDATIVE PHOSPHORYLATION AND METASTASIS IN HEPATOCELLULAR CARCINOMA}

${ }^{1}$ Wang Yilin*, ${ }^{2}$ Wu Weicheng. ${ }^{1}$ Department of Hepatic Surgery, Fudan University Shanghai Cancer Center; Department of Oncology, Shanghai Medical College, Fudan University, China; ${ }^{2}$ Department of Biochemistry and Molecular Biology, School of Basic Medical Sciences, Fudan University, Shanghai, China

\subsection{6/gutjnl-2018-IDDFabstracts.45}

Background Metabolic reprogramming is widely observed in different cancers including Hepatocellular carcinoma (HCC). Reprogrammed metabolism results in hyper-O-GlcNAcylation in tumour cells, which can regulate transcription factors or tumour suppressors to modulate cancer metabolic reprogramming. Rab3A has been reported as an oncogenic factor in some cancers. However, the functions of Rab3A in HCC are never determined. Here we investigated the potential roles of Rab3A in HCC progression and determined how hyper-OGlcNAcylation regulates the functions of Rab3A.

Methods Western blot, qPCR, and immunohistochemistry assays were performed to quantify the relative expression of Rab3A in HCC. The functions of Rab3A in tumour progression were evaluated in HCC cell lines and nude mice. The interaction between Rab3A and OGT was determined by IP and GST pull-down. The GTP-binding affinity was observed in GTP-binding assays. The mitochondrial respiratory capacity was determined by XF cell Mito stress analysis, lactate assays, ROS-Glo H2O2 assays, and MitoSOX assays.

Results Both the mRNA and protein levels of Rab3A were elevated in HCC. However, decreasing Rab3A in HCC cells had no significant effects on tumour progression, and the upregulation of Rab3A in HCC patients conferred no correlations with metastasis or overall survival. We determined that Rab3A is modified with O-GlcNAcylation in HCC, which attenuated the Rab3A-mediated inhibition on HCC metastasis. Further analysis proved that Rab3A and its O-GlcNAcylation also played opposite roles in mitochondria oxidative phosphorylation (mtOXPHOS), and their functions on HCC metastasis partially depended on their effects on metabolic reprogramming.

Conclusions This study evaluated Rab3A as a tumour suppressor in HCC and revealed that the functions of Rab3A in both metastasis and metabolic reprogramming were attenuated by its modification of O-GlcNAcylation in HCC. Mechanistically, Rab3A elevated the expression of some mtOXPHOS-related genes, and O-GlcNAcylation decreased the GTP-binding affinity of Rab3A.

\section{IDDF2018-ABS-0074 CIRCSCD1 PROMOTES FATTY LIVER DISEASE VIA THE JANUS KINASE 2/SIGNAL TRANSDUCER AND ACTIVATOR OF TRANSCRIPTION 5 PATHWAY}

${ }^{1}$ Peifei Li*, ${ }^{1}$ Keshu Shan, ${ }^{1} Y_{i}$ Liu, ${ }^{2} Y u$ Zhang, ${ }^{2}$ Lu Xu, ${ }^{1}$ Lei Xu. ${ }^{1}$ Department of Gastroenterology, Ningbo First Hospital, Ningbo, China; ${ }^{2}$ Ningbo University School of Medicine, Ningbo, China

\subsection{6/gutjnl-2018-IDDFabstracts.46}

Background Non-alcoholic fatty liver disease (NAFLD) is one of the most common liver diseases in affluent countries. Recent studies have reported that circular RNAs (circRNAs) are important regulators of hepatic steatosis. However, the role and mechanism of circRNA in NAFLD are poorly understood.

Methods Through NAFLD-related circRNA microarrays, we used real-time quantitative reverse transcription-polymerase chain reaction to screen circScd1 levels in control and test groups of mice fed a high-fat diet. RNA interference and overexpression plasmid vectors were used to manipulate the expression of circScd1, and the biological effects were evaluated by oil red staining, triglyceride detection, and western blot analysis.

Results CircScd1 expression was significantly lower in NAFLD tissues than in control tissues. Moreover, overexpression of circScd1 significantly inhibited the formation of lipid droplets. Western blot analyses showed that the protein levels of Janus kinase 2 (JAK2) and signal transducer and activator of transcription 5 (STAT5) were significantly increased. However, knockdown of circScd1 significantly promoted the degree of hepatocellular lipidosis and reduced the expression levels of JAK2 and STAT5.

Conclusions Aberrant expression of circScd1 affects the extent of hepatocellular lipidosis in NAFLD and promotes fatty liver disease via the JAK2/STAT5 pathway.

\section{IDDF2018-ABS-0088 ANALYSIS OF CLINICAL SIGNIFICATION KRAS AND SCN5A GENE EXPRESSION IN PANCREATIC CANCER BY TCGA DATASETS}

Xiaobin Shi*. Ningxia Medical University, China

\subsection{6/gutjn|-2018-IDDFabstracts.47}

Background To investigate the prognostic significance of KRAS (Kirsten rat sarcoma viral oncogene) and SCN5A Gene mutation in pancreatic carcinoma by TCGA (the cancer genome atlas) datasets.

Methods The TCGA dataset for liver cancer, gastric cancer, and pancreatic cancer in the TCGA official network, the top 100 genes affected by the number of people affected. Do Wayne map to get genes that do not coincide with the other two diseases in pancreatic cancer, and to find the genetic mutation that has a significant impact on the survival of pancreatic cancer.

Results There are 13329 mutations in pancreatic cancer, and there are 16348 and 19288 mutant genes in the liver and gastric cancer, respectively. The first 100 mutations were analysed from 3 data sets, and the Wayne diagram showed that there are 25 mutations in the mutant gene of pancreatic cancer, the liver cancer and gastric cancer. There are respectively 3 and 11 genes for the coincidence of pancreatic cancer with 